\section{THE CONDITIONS OF ADOLESCENT PATIENTS AT A DANISH TERTIARY UNIVERSITY HOSPITAL}

\author{
K.A. Boisen, P.G. Hertz, C. Blix, G. Teilmann \\ Center of Adolescent Medicine, Copenhagen \\ University Hospital Rigshospitalet, \\ Copenhagen, Denmark
}

Aims: We aimed to explore conditions of adolescent inpatients viewed by the staff.

Methods: Questionnaire survey among the staff at Copenhagen University Hospital Rigshospitalet from five paediatric $(n=36)$ and 13 adult $(n=64)$ inpatient wards.

Results: Confidentiality and consent: Only $1 / 3$ of the participants estimated that their colleagues were fully familiar with the rules on confidentiality and consent for adolescents $<18$ years.

Staff skills: $28 \%$ knew of professionals with special skills in the care of adolescent patients. However, there was no structured education or evaluation. Only a small minority knew any written guidelines for the care of adolescent patients.

Involvement of adolescents: $70 \%$ found it important to involve young patients when deciding whether admission should take place at the paediatric or adult ward. However, this was the case in only a minority of cases. At the paediatric departments, $1 / 4$ of the staff felt that the patients were involved too little in treatment decisions.

Environment: Dayrooms dedicated for adolescents were present in two of the paediatric and none of the adult wards, and only $1 / 3$ of staff found that the general facilities met the need of adolescents. There were activities dedicated for adolescents at two paediatric and one adult department. Conclusions: Although international guidelines for adolescent friendly health services exist, there was no structured staff education or clear written guidelines. Involvement of adolescent patients regarding treatment decisions could be improved especially at the paediatric departments. Adolescent-specific activities and environment were sparse especially at the adult wards.

\section{PRETERM-BORN INFANTS PRODUCE LESS MELATONIN THAN TERM-BORN INFANTS AT 9 AND 12 MONTHS OF AGE: A RANDOMIZED CONTROLLED TRIAL}

\author{
S. Goldstein ${ }^{1}$, I.R. Makhoul ${ }^{2}$
}

${ }^{1}$ Neurobiochemisty, Tel-Aviv University, Tel Aviv, ${ }^{2}$ Neonatology, Rambam Medical Center, Haifa, Israel

Background: Melatonin production is known to be delayed in preterm-born infants up to 6 months of age. We aimed to test the profile of melatonin production in these infants at 9-12 months of age.

Methods: Twenty three term-born and 23 pretermborn infants (gestational age: 29-34 weeks) were randomly assigned. We tested nocturnal urinary melatonin excretion, within a repeated measures design, both at 9 and 12 months of age. Nocturnal urine was extracted from diapers and urinary melatonin derivate (6-sulphatoxymelatonin) excretion was analyzed by ELISA assay.

Results: Preterm-born infants had significantly lower urinary melatonin excretion both at 9 and 12 months of age as compared to term-born infants. At 9 months: $8502 \pm 6215$ vs $12735 \pm 10284 \mathrm{ng}$, and at 12 months: $8623 \pm 5095$ vs $14985 \pm 10890 \mathrm{ng}$ respectively, $[F(1,42)=4.276],(p<0.05)$.

Conclusion: At 9 and 12 months of age, the delayed maturation of melatonin production is still persistent in preterm-born infants as compared to term-born mates. The impact of persistent decreased melatonin production in preterm-born infants up to 12 months of age on their psychomotor development is to be elucidated. 